DOI: 10.53464/JMTE.02.2021.08

Journal of Marine Technology and Environment Year 2021, Vol.II

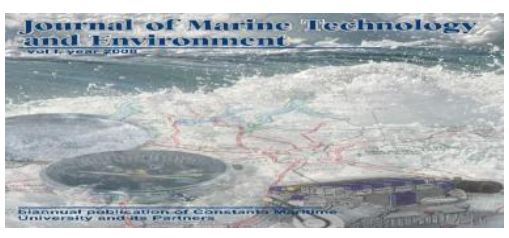

\title{
WASTE MANAGEMENT ON BOARD SEAGOING SHIPS
}

\author{
Liliana-Viorica Popa \\ Constanta Maritime University, Faculty of Navigation, 104 Mircea cel Batran Street, 900663, Constanta, Romania, e-mail: \\ lili_vio@yahoo.co.uk
}

\begin{abstract}
The reality remains uncomfortable, the climate crisis is coming with extreme phenomena more and more devastating around the globe and more and more people and more and more parts of ecosystems are suffering from the greed of large companies and lack of constructive action and coherent decision-makers.

There are various types of waste generated during operation, and to be able to implement a waste management system, it is necessary to identify and quantify the different types of waste on board.

The purpose of this paper is to examine the responsibilities, management, handling and disposal of ship-generated waste and the requirements for the preparation of an on-board waste management plan.

Key words: garbage, disposal, management plan, responsability, pollution, prevention, segregation.
\end{abstract}

\section{INTRODUCTION AND OBJECTIVES}

Garbage management needs to be undertaken on board in order to prevent garbage entering the marine environment or from causing unnecessary risks to health and safety on board.

- $\quad$ Some items discharged overboard take extremely long time to decompose, and remain in the marine environment for extended periods.

- Marine animals can sometimes mistake garbage items for food, and become entangled in the item, or die of poisoning or suffocation and ingestion

- $\quad$ Rubbish washed up ashore looks unsightly

and may cause unpleasant odours, and may pose hazards to beach / inshore water users

- Ineffective garbage management on board can result in a health and safety risk to crew.

One of the important regulations on the topic of the paper is represented by MARPOL Annex V, Regulation 10 (2)[1]

"Every ship of 100 tons gross tonnage and above, and every ship which is certified to carry 15 persons or more, and fixed or floating platforms shall carry a garbage management plan which the crew shall follow. This plan shall provide written procedures for minimizing, collecting, storing, processing and disposing of garbage, including the use of the equipment on board. It shall also designate the person or persons in charge of carrying out the plan. Such a plan shall be in accordance with the guidelines developed by the Organization and written in the working language of the crew."[2]

It should be noted that, whilst it is a mandatory requirement to have on board a garbage management plan, this plan need not be approved by the Administration.

All vessels are required to conform with the Company's Garbage Management Plan [3].

The following information is designed to inform of the key requirements and relevant considerations so that safe and efficient garbage management arrangements can be devised and implemented on board.

In order to comply with environmental requirements, each shipping company must implement a waste management plan.

The objectives of this plan are: 
DOI: 10.53464/JMTE.02.2021.08

- To provide managed vessels with a plan that meets the requirements of MARPOL Annex V Regulation 10 (2) and, facilitates overall compliance with the requirements of MARPOL and other relevant legislation concerning the management of shipboard generated waste.

- Provide a plan to enable implementation and achievement Environmental Protection Policy.

\section{KEY RESPONSIBILITIES}

\subsection{Designated Person on board-Chief Officer}

The Duties of the Chief Officer (Designated Person) will include:

- $\quad$ Ensuring placards are displayed in accordance with the regulations

- $\quad$ Ensuring that personnel are fully familiar with, and comply with the Ship's Garbage Management Plan, including full familiarization and introductions for new joining personnel, the locations of the Special Areas designated under MARPOL regulations, in which absolutely no discharge overboard is permitted.

- $\quad$ Ensuring that the persons responsible for garbage collection, segregation and management, receive the appropriate training and are capable of managing the operation proficiently.

- $\quad$ Ensuring that sufficient numbers of crew are responsible to work in line with the ship's requirements.

- $\quad$ Ensuring incineration or other treatment of garbage in accordance with the instructions.

- $\quad$ Ensuring that all garbage is being stored in a safe and hygienic manner.

- $\quad$ Liaising with shore authorities for port

reception facilities and details of any restrictions on the use of incinerators in port.

- $\quad$ Liaising with Master and Chief Engineer on a daily basis regarding any problems encountered with garbage management.

- $\quad$ Reviewing garbage management practices on board the ship and recommending amendments to the plan as necessary.

- $\quad$ Ensuring that the Garbage Record Book is completed and signed as required by the regulations and the instructions of the book.

- $\quad$ Ensuring that the Bridge is informed of any disposal to sea, so that the correct position can be determined and recorded in the Garbage Record Book.

- Confirming with any relevant department on board and ashore if garbage collection and sorting procedures are not in line with this policy

- The Designated Person, along with the

Environmental Officer, should ensure that garbage management on board is suitable to meet the requirements. Additionally, the Master shall satisfy himself during the course of weekly vessel inspections that the requirements of this Plan are being met and that garbage management on board is in compliance with the regulations.

\subsection{All Crew}

Assistance to the Chief Officer and Environmental Officer as requirement to ensure that the minimization, collection separation and processing of garbage is appropriate and efficient in all areas of the ship.

Crew members responsible for specific areas [3]:

- $\quad$ Deck Department - Duty A/B (as nominated by $\mathrm{BSN}$ or $\mathrm{C} / \mathrm{O}$ )

- $\quad$ Engine Department - Duty M/M and/or OILER (as nominated by $1 / \mathrm{E}$ )

- Galley/Accommodation - Steward/Messman

Personnel as designated above will be responsible for the inspection and collection of the garbage receptacles in their area, and delivery to the ship's central collection point as per the following schedule:

- $\quad$ Navigation Bridge - DAILY

- Galley - 3 x PER DAY

- Officer Pantry - 3 x PER DAY

- $\quad$ Crew Pantry - 3 x PER DAY

- $\quad$ Engine Room - Control Room - DAILY

- $\quad$ Engine Room - Workshop - DAILY

\subsection{Training and Familiarization (Part of VQS21 Vessel} Familiarization)

- All personnel should be trained to recognize different garbage types.

- All personnel should be aware of the importance of segregation at the point of collection.

- $\quad$ All joining crew members should be informed that discharge overboard of garbage other than food waste is not permitted under MARPOL ANNEX $\mathrm{V}$ and that no garbage should be discharged overboard without permission of the Bridge /OOW [3].

- All personnel should be trained on the requirements relating to MARPOL Annex V Special Areas. 
DOI: 10.53464/JMTE.02.2021.08

- $\quad$ New Joiners should be informed of EPS targets related to garbage disposal, and updated on the ship's progress in achieving them.

- All Personnel are required to participate in the garbage management system as specified in this plan.

- Any Personnel who will be using processing equipment (incinerators, compactors, grinders) should be competent in their use, including the use of appropriate PPE.

Thereafter, all personnel on board will need to be familiarized with:

receptacles

- Any changes to required sorting at garbage collection points

- $\quad$ Any changes to collection procedures, processing procedures and storage facilities

- The changes to the overboard disposal requirements.

\section{CATEGORIZATION OF GARBAGE}

Garbage is to be grouped into categories for the purposes of recording in parts I and II of the Garbage Record Book (or ship's official log-book) as follows:

\section{Part I}

- A - Plastics

- B - Food wastes

- C - Domestic wastes

- D - Cooking oil

- E - Incinerator ashes

- F - Operational wastes

- G - Animal carcasses

- $\mathrm{H}$ - Fishing gear

Part II

- I - E-waste

- $\mathrm{J}$ - Cargo residues (non-HME)

- $\mathrm{K}$ - Cargo residues (HME)

\section{GARBAGE RECORD BOOK}

The Master and $\mathrm{C} / \mathrm{O}$ should be familiar with making and signing off entries in the Garbage Record Book.

All activities in relation to the disposal of garbage ashore, overboard or via the incinerator, should be recorded in the ship's garbage record book and authorized by the Chief Officer. Any discharge whilst in port, should be confirmed with port staff/shore personnel in advance of the discharge. Reconfirmation details should also be recorded in the Record Book.

Garbage Record Books should be retained on board from the date of the last entry, for two (02) additional years.

Garbage Record Books will be inspected by the company's auditors, and non-conformities or observations will be raised accordingly and followed up by HSE Department [3].

Garbage Record Books will also be required for review during internal and external ISO 14001 Environmental Management Audits [4].

Offlanding of dunnage and packaging materials received during bulk supplies of stores / spares should also be recorded in the Garbage Record book. This can be recorded under Category A, Category $\mathrm{C}$ or Category $\mathrm{F}$ as applicable with suitable remarks. Every effort should be made to obtain receipt of off landing from the stores / spares supplier, however where this cannot be achieved, a self-declared statement shall be made and appended to the Garbage Record Book.

\section{SHIPBOARD GARBAGE HANDLING (PLANNING)}

In order to implement a garbage management plan on board which will comply with the regulations, the following points will need to be taken into account:

1. Vessels should have a designated garbage storage room / area. This should be easily accessible from all other parts of the ship via short access routes but generally away from the accommodation areas. Refrigeration may also be useful for storing food wastes, and note that some wastes may need to be stored for a long time [5].

2. Garbage receptacle stations should be located separately from accommodation areas, but still easily accessible for crew members to use. Additionally, they should be located such that disposal to shore is a short journey not through accommodation.

Garbage receptacles should be colour-coded as follows (Figure 1)[6]:

- PLASTICS - (Red)

- FOOD WASTE - (Green)

- OTHERS - (Blue)

(Domestic \& Operational Waste)

- INCINERATOR ASHES - (Black)

- BATTERIES, LAMPS - (Grey) 
DOI: 10.53464/JMTE.02.2021.08

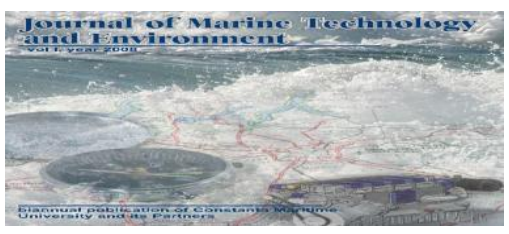

- E WASTE - (White)

- OILY RAGS - (Black with White band)

- BIO FOULING WASTE - (Yellow with Black Band)

- CARGO RESIDUES (HME) - (Grey with White band)

(Applicable only to Bulk Carriers Only)

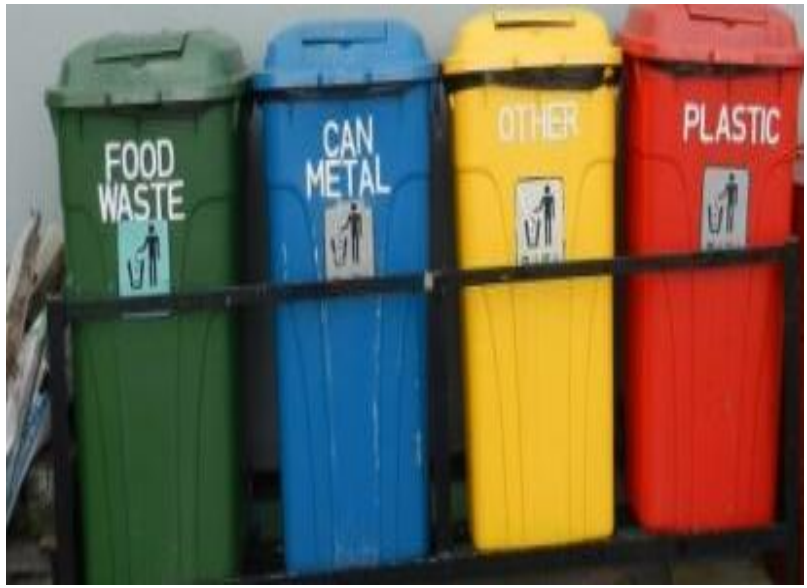

Figure 1 Garbage colour coded [6]

3. Remember that plastic items will need to be kept separate, and any incinerator ash will have to be stored on board for disposal ashore. No plastics, plastic ash or plastic residues may be discharged overboard.

4. Recyclables should be kept separately as far as possible, so that they may be taken ashore and recycled (funds raised maybe placed into crew mess funds). Vessels are encouraged to use recycling facilities when they are available, and to report to Safety Department any particularly good ports with regards to recycling services.

5. Contaminated food waste, or items contaminated with such wastes may require special handling procedures, including refrigeration.

6. Placards clearly demonstrating garbage collection for each bin/receptacle should be used. Placards should contain a summary declaration stating the prohibition and restrictions for discharging garbage from ships under MARPOL Annex V. Placards should be easy to understand and use pictures to clearly identify what garbage should be placed in what bin. They should be displayed at eye level in areas where garbage is collected e.g. mess rooms, galley, and bridge (Figure 2)[7].

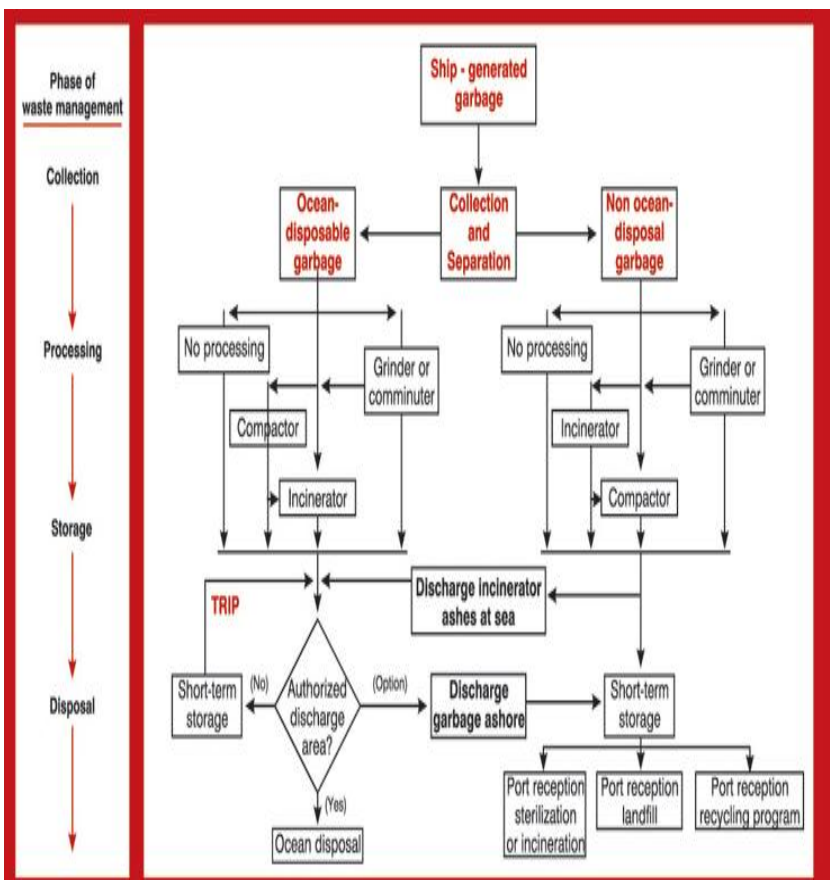

Figure 2 Shipboard handling and disposal of garbage [7]

7. These placards should be $12.5 \mathrm{~cm} \times 20 \mathrm{~cm}$, made of durable material and fixed in conspicuous places on board the ship (Figure 3) [8]. Placards should be replaced if damage or wear compromises the readability.

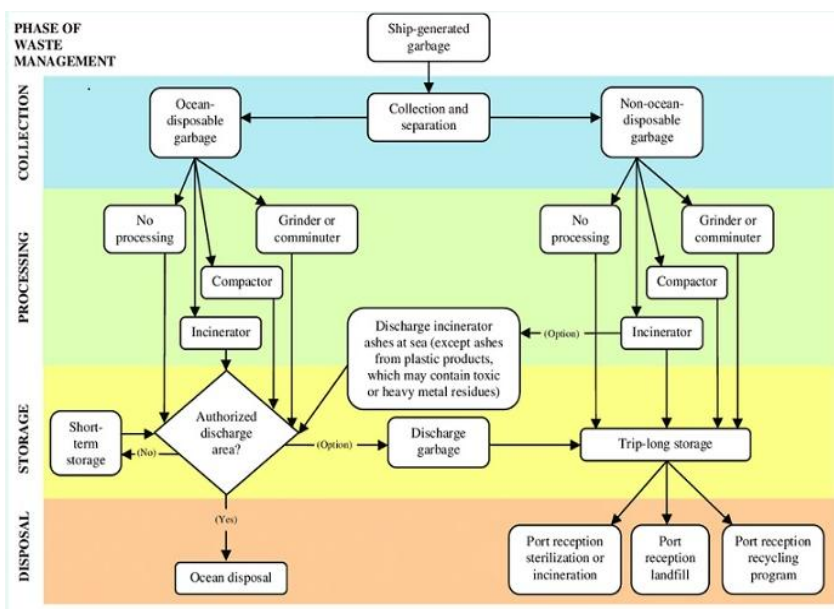

Figure 3 Waste management onboard [8] 
8. When planning garbage handling for the duration of the coming voyage, due attention should be paid to: between port calls

- $\quad$ Number of crew on board.

- $\quad$ Facilities on board for waste management storage, compaction, grinding, incineration.

- Number of cargo operations expected (significant volumes of garbage are generated from port-cargo operations).

- Available facilities in each location (including potential to utilize free services or recycling facilities).

Any specific local restrictions which may impact upon final garbage disposal plan for the voyage.

- $\quad$ Any sanitary or safety risks of the garbage or wastes to be stored.

\section{GARBAGE MINIMAZATION}

Ships should try to reduce the amount of material taken on board that will eventually become garbage. Some possible actions are:

o Reject excessive packaging for shops and spare parts delivered on board;

o If suppliers cannot remove excessive packaging, inform the Purchasing Department. or include comments in the supplier evaluation [9];

- Ships should also try to minimize on-board garbage production. To this end, on-board staff should [10]:

o Minimize the generation of garbage on board, e.g. reducing the printing / printing requirements on both sides of a page;

o Consider whether an item can be REUSED before disposal or RECYCLED instead of disposal;

o Use storage systems and methods for reusing drainage, support, lining and packaging materials

o Use the unloading in the port reception facilities of lining and packaging materials generated in the port during loading activities, as discharge into the sea is not permitted. Ships should try to reduce the amount of material taken on board that will eventually become garbage. Some possible actions are:

o Reject excessive packaging for shops and spare parts delivered on board;

o If suppliers cannot remove excessive packaging, inform the Purchasing Department. or include comments in the supplier evaluation;
- Ships should also try to minimize on-board garbage production. To this end, on-board staff should:

o Minimize the generation of garbage on board, e.g. reducing the printing / printing requirements on both sides of a page;

o Consider whether an item can be REUSED before disposal or RECYCLED instead of disposal;

o Use storage systems and methods for reusing drainage, support, lining and packaging materials;

o Use the unloading in the port reception facilities of lining and packaging materials generated in the port during loading activities, as discharge into the sea is not permitted [11], [12], [13], [14].

\section{CONCLUSIONS}

All garbage generated on board should be collected at designated garbage collection points. No garbage may be immediately thrown overboard [10].

All garbage should be sorted in the Central Garbage Collection Point and segregated according to the requirements as documented in this plan.

Any food waste which is to be disposed of to sea must be done so with the permission of the $\mathrm{C} / \mathrm{O}$ or OOW, and appropriately recorded in the Garbage Record Book part I.

Any Cargo Residues which are to be disposed at sea must be done so with the permission of the $\mathrm{C} / \mathrm{O}$, only the residues that are not harmful to marine environment (nonHME) are permitted to be discharged at sea as per regulation 4 and 6 of MARPOL Annex V.

The disposal should be appropriately recorded in the Garbage Record Book part II.

All other garbage should be either discharged to shore, or incinerated accordingly.

\section{REFERENCES}

[1] MEPC Resolution 219 (63) / 2012, Guide for the implementation of Annex V to Marpol, revised

[2] MEPC Resolution 220 (63) / 2012 - Guide for the elaboration of ship management plans on board ships

[3] Amendments to the Annex to the 1978 Protocol to the 1973 International Convention for the Prevention of Pollution from Ships, adopted by the International Maritime Organization by Resolution of the Committee for the Protection of the Marine Environment - MEPC. 89 (45) in London on 5 October 2000 
DOI: 10.53464/JMTE.02.2021.08

[4] Order of the Minister of Transports, Constructions and Tourism no. 322/2006 with the subsequent modifications and completions regarding the port installations for taking over the waste generated by the ships and the cargo residues

[5] GD 856/2002 on the record of waste management and for the approval of the list containing waste, including hazardous waste.

[6] https://maritimecyprus.com/2017/08/24/amendmentsfor-garbage-management-plan-and-garbage-record-bookcoming-into-force-march-01-2018/

[7]

https://www.lalizasimosigns.com/product.php?selected_id $=4128$

[8] http://shipsbusiness.com/garbage-collection.html

[9] Government Decision no. 349/2005 regarding waste storage.

[10] OUG 195/2005 regarding environmental protection

[11] OUG 71/2010 regarding establishing a strategy for the marine environment.

[12] Sundri Iuliana, 2015, Ecotoxicological assessment in some points of the ROMANIAN BLACK SEA coastal zone, Journal of Marine Technology and Environment, vol.II, pp 79-82.

[13] The Commision on the Protection of the Black Sea Against Pollution, 2009.

[14] www.blackseacommision.org/main.asp 